Research Article

\title{
CPLD-Based Displacement Measurement System for Nanoscale Grating Ruler
}

\author{
Jiyuan Sun ${ }^{1,2,3}$ and Chunlin Tian (D) ${ }^{1,4,5}$ \\ ${ }^{1}$ College of Mechanical and Electrical Engineering, Changchun University of Science and Technology, Changchun 130022, \\ Jilin, China \\ ${ }^{2}$ College of Electrical and Information Engineering, Beihua University, Jilin 132021, Jilin, China \\ ${ }^{3}$ Jilin Guangyu Technology Co., Ltd., Jilin 132021, Jilin, China \\ ${ }^{4}$ Guangdong Guangji Hi Tech Co., Ltd., Foshan 528000, Guangdong, China \\ ${ }^{5}$ Changchun University of Science and Technology, Chongqing Research Institute, Chongqing 401135, Chongqing, China
}

Correspondence should be addressed to Chunlin Tian; tcl@cust.edu.cn

Received 21 July 2020; Revised 29 August 2020; Accepted 14 October 2020; Published 3 November 2020

Academic Editor: Tifeng Jiao

Copyright (c) 2020 Jiyuan Sun and Chunlin Tian. This is an open access article distributed under the Creative Commons Attribution License, which permits unrestricted use, distribution, and reproduction in any medium, provided the original work is properly cited.

\begin{abstract}
With the continuous development of science and technology, industrial production has higher and higher requirements for precision. Many high-precision measurement technologies emerge as the times require, and nanoscale grating ruler displacement measurement technology is one of them. As a kind of precision sensor, nanoscale grating ruler has important application in displacement measurement system. CPLD has the advantages of high integration and fast programming speed, which is often used to control the displacement measurement system of nanoscale grating ruler. The purpose of this paper is to deeply explore the measurement effect and related application principle of nanoscale grating ruler displacement measurement system based on CPLD technology. A set of nanoscale grating ruler displacement measurement system is designed based on CPLD technology. The output signal of grating ruler is programmed by CPLD. The $x$-axis displacement of the experimental platform controlled by stepping motor is measured, and the measured data are recorded by carrying out analysis and research. The results show that compared with the traditional phase difference measurement system, the measurement accuracy of the system based on CPLD is improved by $24.7 \%$, the robustness of the measurement system is improved by $18.6 \%$, and the measurement speed is increased by $27.3 \%$. Therefore, this kind of nanoscale measurement precision grating ruler displacement measurement control system based on CPLD has three characteristics: high measurement accuracy, strong anti-interference ability, and high measurement motion efficiency, which can effectively meet the requirements of grating ruler displacement measurement system for high-precision manufacturing technology.
\end{abstract}

\section{Introduction}

Manufacturing is already a major pillar industry of a country, and it is the most fundamental technological foundation on which a country depends for prosperity. At present, manufacturing engineering science and mechanical manufacturing engineering technology are rapidly developing towards the two directions of ultraprecision and miniaturization. Ultraprecision micromachining has developed into one of the important technical contents of advanced machinery manufacturing [1]. At present, the processing of superfine materials has gradually entered a new field of nanotechnology. Grating measurement displacement technology with higher nanometer order and high resolution is already an indispensable important part in the processing of modern superfine materials, and it is also urgently needed. One of the three key technologies is studied and solved [2]. Compared with other linear and displacement measuring sensors, the grating ruler has a high technical comprehensive application advantage in improving the measurement digital display accuracy, resolution, reliability, technical requirements for the application environment, and prices. Therefore, the use of grating rulers has a very wide range of applications in 
measuring digital displays, CNC machine tools, and measuring instruments [3].

Nanometer-scale grating interferometric measurement technology mainly uses interference diffraction-type gratings. The grating pitch is generally $2 \mu \mathrm{m}$ or $0.5 \mu \mathrm{m}$. The diffraction width of the grating line is close to the diffraction wavelength of light, which can produce interference diffraction and short-wave interference luminescence. The phenomenon and so on forms fringes, and its principle for measuring light is also called the principle of optical interference diffraction [4]. In view of the shortcomings of the control accuracy of the traditional standard scale displacement measurement and management system, this paper proposes a CPLD-based two nanometer-scale displacement measurement control system for standard grating size, which is used in precision instrument industry production and testing equipment. In the scale mobile sensor, CPLD has many advantages such as high system integration, fast working speed, convenient programming, low price, and so on [5]. On the basis of optimizing and improving the key technology of the displacement measurement system of the nanoscale grating ruler, using CPLD to assist the development can not only improve the accuracy, integration, and reliability of the displacement measurement system, but also reduce the production cost of the displacement sensor of the nanoscale grating ruler.

In order to explore the measurement effect and related application principles of the CPLD-based nanoscale displacement measurement system, this paper consults a large number of related materials. Among them, Versino et al. introduced the existing displacement measurement system in China and emphasized that the existing relatively backward displacement measurement technology could not meet the industrial production requirements of high-precision requirements and analyzed the current research status and research of nanoscale grating rulers in China significance and put forward the technical guidance and research direction of nanoscale grating ruler [6]. Xu et al. gave a detailed introduction to the principles and applications of nanotechnology, carefully discussed the core theories of nanotechnology, pointed out that the emergence of nanotechnology is a huge industrial change, and affirmed the importance of nanotechnology, but also pointed out that nanotechnology. The shortcomings of technology and the points of attention in application are shown in [7]. Peipei et al. elaborated the development process and application field of CPLD in detail, discussed the working principle of CPLD, and summarized a set of CPLD programming control technology, and the experiment proved the feasibility of CPLD programming control technology [8]. Pinna et al. designed a CPLDbased scale displacement measurement system for nanoscale gratings, improved the design ideas, and made a detailed introduction to the working principle of this scale displacement measurement system. This design is still in the testing stage [9]. Through research, Wang et al. found that the CPLD-based nanoscale displacement measurement system has the characteristics of high measurement accuracy, convenient operation and practicality, high system robustness, and high detection efficiency. It is a displacement measurement technology with broad application prospects [10].

\section{Purpose}

The purpose of this article is to explore the measurement effect and related application principles of the CPLD-based nanoscale displacement measurement system. On the basis of summing up the precious experience of previous people, this paper has made relevant improvements in research methods and design schemes. First of all, this article uses CPLD as the technical basis for the measurement of the zeropoint displacement of various nanotechnical-grade solid grating sizes and provides the zero-point measurement temperature reference directly in the form of physical software during the design of the system software, using a solid with low hot spots and expansion temperature coefficient. Nanogratings are made directly from solid materials such as quartz or zero-point thermal expansion coefficient glass. Secondly, this article takes the multimeasurement grating system as the technical basis, adopts the principle of the new static grating error parameter corrector, and based on the research of the current measurement results, the multimeasurement grating system as the technical basis is based on the secondary. A new type of high-precision grating measurement sensor for measuring nanoparameter gratings of moiré fringe diffraction signals.

\section{Working Principle of Grating Ruler}

The basic principle of the design of the displacement signal measurement and processing system on the grating ruler is mainly to use the relative direction between the scale and the raster scanning image mask to move to form moiré fringes under the illumination of the incident light source [11]. The moiré fringes pass through multiple photoelectric. After the signal sensor is converted, it becomes an approximate sine and cosine wave photoelectric signal, which is the original raster scanning mask signal [12]. Then we use different pulse tube fine analysis methods to obtain different automatic counters and pulse metering signals for the measuring device and step distance [13]. The pulse count signal is generally considered to be a continuous twopath orthogonal pulse signal. The two pulse signals are, respectively, connected to the subsequent two-way reversible automatic counting control circuit, the calculated parameter value of the counter is multiplied by the value, and the step of the measuring device is the radial displacement of the measuring scale and its measured count value [14]. The maximum speed of the grating ruler movement measurement allowed signal movement cycle speed is generally determined by the measurement grating ruler input and output movement frequency and the input signal movement cycle frequency of the raster scan image signal. The calculation formula of the maximum displacement speed of the grating ruler measurement is shown in the following equation: 


$$
L=\sum_{J+1}^{V-N} \frac{D_{f}}{D} * \operatorname{Info}\left(D_{f}\right) .
$$

Among them, $L$ represents the maximum displacement speed of the grating ruler; $D$ represents the output frequency.

Under the certain premise that the movement signal oscillation period of the measured signal of the grating is unchanged, vamp is proportional to its frequency at the maximum output of the grating ruler, and the step speed during measurement corresponds to its resolution. If we increase the current electronic measurement [15], the electronic subdivision multiple and frequency of the subdivision counting circuit will also increase its resolution (due to the reduction in step speed during measurement), because we are subject to the maximum response output frequency of the current electronic metering subdivision counting circuit and for subsequent. The maximum input response frequency of the electronic counting subdivision circuit is very limited. With the continuous increase of the electronic subdivision frequency multiples, the maximum output response frequency of the grating ruler will also decrease, so the maximum measurement speed may allow the measurement speed to decrease. Therefore, when the maximum allowable speed is measured, the maximum measuring speed step of grating ruler is directly proportional to the positive and negative proportion of the maximum grating speed. Therefore, the nanoscale ruler is used for high-speed movement measurement (the maximum speed when moving at the allowed speed is generally $\geq 10 \mathrm{~m} / \mathrm{s}$ ), the movement resolution is generally set to the order of nanometers $(0.3 \sim 1 \mu \mathrm{m})$, and if used for nanometer scales with low-speed movement measurement (movement speed is generally $\leq 200 \mathrm{~mm} / \mathrm{s}$ ), the resolution is likely to reach the order of micro-nano $(1 \sim 30 \mathrm{~nm})$.

\section{Signal Switching Synthesis of Grating Ruler}

The displacement measurement in the above cases is one of the advanced grid rulers (coarse grid ruler) suitable for lowresolution and high-speed measurement in the fast startup state, and the other is the ordinary nano or advanced standard grid ruler (fine grid ruler). When the positioning motion control process of the detection system changes from fast motion to medium motion to slow motion, when the axis of the detection system starts to move at high speed, the number of pulses is generated by the fast switching motion and the number of pulses of the nanoscale scientific ruler [16]. The coarse and fine optical rulers simultaneously measure the movement of the system axis and the displacement velocity at the beginning of the movement [17]. An important characteristic of a single fine grid ruler is that the maximum speed allows the system to move and the measurement speed is proportional to the maximum resolution. Therefore, the measurement speed vim exceeds the maximum speed of the fine grid ruler during the measurement period 1-2 and the system deceleration movement [18]. During the measurement of the speed Vmax, the system will decelerate. During the measurement period 3, the other measurement indication signals on the coarse fiber scale are valid, and the other measurement indication signals on the fine grid ruler are valid and invalid [19]. When the Vmax speed in period 3 is decelerated, the pulse signal of the displacement gauge and the roughness scale is used as the signal of the maximum displacement characteristic measurement degree [20]. In addition, since the displacement characteristic of acceleration enables speed movement, the maximum allowable measurement speed and the measurement moving speed $(>1 \mathrm{~m} / \mathrm{s})$ at full scale can be obtained [21]. When a chemical system starts to move slowly and continuously at a certain time (in the vtype 1 nanometer chemical lattice ruler, the counter and pulse are switched quickly, and this process is actually at a certain moment) (the chemical lattice ruler counter is pulse edge, the moment when the zero point rises rapidly), a rapid movement clears the switching process between the precise grating ruler and the counter. The counting pulse calculation formula of the nanoscale grating ruler is shown in the following equation:

$$
f=\iint_{\Omega}\left(y^{2}+M^{2}\right) p d M .
$$

Among them, $f$ represents the pulse number of the nanoscale grating ruler; $M$ represents the grating ruler grid number.

\section{Design of Measurement System}

In this paper, the CPLD2018 chip is mainly used to subdivide the direction output control signal circuit of a nano-quality conductor grating ruler [23]. According to the directional phase shift relationship between the two orthogonal direction encoding control signals, the two orthogonal inputs of the chip are encoded. The signal is converted into two control signals for output: one signal output is the four-bit triple frequency control signal after the object is subdivided, and the other signal output is the direction control signal when the object moves [24]. After the fine resolution, the signal with nano power level and grating size will be connected to the microcontroller; that is, the two cent pins of a CPLD2018 are connected to the cnp2 port of the automatic controller. The signal parameters are synchronously counted and automatically calculated and processed to obtain an actual displacement in the $x$ - and $y$-directions. That is, when an $x$-direction two-axis edge-finding motor can drive the two-axis edge-finding device to move on an $x$-direction axis, if a touch contact encounters a passively detected object, the motor generates a control signal to output, the controller [25]. After detecting and receiving the signal input by the motor, a corresponding control action and data processing can be performed. This control system mainly adopts the photoelectric driven inductive workpiece edge finder, using the conductive motion characteristics of an object of a workpiece. When a trigger probe directly touches the object to the surface of a workpiece object, an inductive loop is formed on the circuit, which generates a contact output electrical signal. Using these basic characteristics of the automatic edge finder, it is fixed on the $y$-shaped axis, and 
the wireless contact signal is obtained to insert the output trigger signal to the edge of the surface of the automatic workpiece object, forming the edge output trigger signal input of the automatic controller. In the structural design of the control system, the control management system realizes the transmission of control data packets and the reception of the control system commands to the transmitter through the serial bus communication. There is a half-port or full-duplex single-port serial data communication port inside the controller, so a serial data communication can also be conveniently carried out between a controller and two upper computers at the same time. Before the motor performs a serial communication, it must meet certain voltage conditions, because the parallel serial port voltage of the cold machine is higher than the rs220 voltage level and the parallel serial port tbl20 level of the single-chip computer, so they must be between them. There is a circuit connected to the serial converter above the voltage level. The automatic collection of the output data of the automatic displacement of a nanoscale microscale is to collect the signal data output by the displacement of a nanoscale microscale through the fine-grain resolution. Since a nanometer-size level is a physical period of the output signal of the grating size, it represents a displacement of $4 \mu \mathrm{m}$, and the cycle of each point of the output signal after fine resolution represents a displacement of $2 \mu \mathrm{m}$. After calculating the value of the counter in the controller, it is displacement data. The numerical calculation formula of the counter of the nanoscale grating ruler displacement measurement system is shown in the following equation:

$$
N(c)=\left(S_{k}-T_{k}\right) * F_{k} *\left(N-c_{k}\right) k^{N},
$$

where $N(c)$ represents the value of the grating counter, and $N$ represents the calculation period coefficient of the grating.

\section{Implementation of Measurement System Based on CPLD}

The biggest feature of the nanoscale grating displacement measurement system designed in this paper is that except for some peripheral analog circuits, the digital circuits involved are all realized by CPLD. Each part of the circuit is designed and compiled in the CPLD development software MAX + PLUS II to generate programming files and then downloaded to the CPLD chip. The two same-frequency signals FR and FS output the phase difference signal phase through the CPLD phase detector. The phase signal is sent to the gate of the counter as the gate signal to control the counting time of the counter, and its function is equivalent to the gate circuit. The input pulse reference count signal is multiplied by the pulse frequency multiplier and used as a pulse counter. The counter pulse count signal pulse is input to CLK, and at the same time it is sent to the integrated control processing link for control conversion. Because the grating rule requires a total of 1200 pulse signals throughout, CPLD internally designs an 18-bit reversible counter; the lower 10 bits represent data, the highest bit is the status bit, and the display result is 1 , indicating that the current position of the grating ruler is in the negative direction of the initial zero point. If it is 0 , it means that the current position of the nanoscale grating ruler is the square of the initial zero point. The three pairs of square wave signals output by 4006 after being shaped by $90^{\circ}$ phase difference are A1 and B1, A2 and $\mathrm{B} 2, \mathrm{~A} 3$ and $\mathrm{B} 3$, respectively. When the A-channel signal in CPLD has an upward edge, the B-channel signal is detected. If the B-channel signal is high, it indicates that the nanoscale grating ruler is moving in the positive direction, and the current counter increases by "1." If the signal of channel B is low, it means that the nanoscale grating ruler is moving in the negative direction, and the current counter is decremented by "1." Due to the high efficiency, signal-tonoise ratio, and other photoelectric signal characteristics of the photoelectric converter using clad two chips, it can only directly reach the optimal value required by the process performance design of the related device system under the appropriate operating timing conditions, and the output performance is stable reliable photoelectric signal; the process design of the driving integrated circuit has therefore become one of the key issues in the process design process. In this paper, the external pulse filter signal is shaped by 4006 trigger pulse filter and then sent to the cold for the feeder. The CPLD uses the CPLD to count and calculate the external pulse filter signal and store the pulse data in the internal pulse register. The single chip microcomputer reads the data quickly through a bus data interface, transforms the scaled function of the input data frequency, and sends it to the bus LCD line for precision display. The working parameters of the CPLD chip in the measurement system designed in this paper are shown in Table 1.

\section{Verification Results and Related Discussions}

7.1. A Statistical Result of Displacement Measurement Speed and Accuracy of Nanoscale Grating Ruler. Using CPLDbased displacement measurement system of nanoscale grating, the principle verification experiment was carried out on the displacement measurement system. The comparison results of the measurement data show that the displacement measurement system of the nanoscale grating and the displacement measurement system of the commercial laser interferometer can describe the movement of the moving stage. As the displacement of the moving stage increases, the displacement difference between the two measurement systems gradually increases. This is caused by the cosine error caused by the angle between the measuring direction of the nanoscale or laser interferometer and the moving direction of the moving table. Statistical analysis shows that the correlation coefficient of the measured data of the two displacement measurement systems is 0.8627 , and the experiment verifies the correctness of the principle of the nanoscale displacement measurement system. The cosine error of the displacement measuring system of the nanoscale grating ruler based on CPLD is shown in Table 2 .

Through the analysis of the displacement measurement system based on CPLD-based nanoscale ruler and the displacement measurement system of laser interferometer on the displacement measurement of the moving platform, the 
TABLE 1: CPLD chip working parameters.

\begin{tabular}{lccc}
\hline Model & Basic materials & Signal period & Frequency \\
\hline Test deployment & Ceramics & 0.25 & -1000 \\
Voltage & Aluminum alloy & 0.33 & -850 \\
Electric current & Copper alloy & 0.47 & -1200 \\
Power & Glass & 0.59 & -1130 \\
Length & Steel belt & 0.84 & -1480 \\
\hline
\end{tabular}

TABLE 2: Cosine error of displacement measurement system of nanoscale grating ruler.

\begin{tabular}{lcccc}
\hline Group & Collection speed & Coarse grating & Fine grating & Switch synthesis (\%) \\
\hline Operating cycle & 165.7 & 55.34 & 338.7 & 19.5 \\
Operating hours & 215.3 & 62.88 & 426.1 & 36.7 \\
Large range & 185.6 & 71.25 & 297.4 & 65.3 \\
Cosine error & 203.8 & 81.61 & 358.2 & 75.1 \\
\hline
\end{tabular}

measurement consistency experiment was carried out. The CPLD controls the grating ruler to make periodic reciprocating movements in the measurement direction, and at the same time the grating ruler and the laser interferometer displacement measuring system measure the displacement of the moving table at a frequency of $5 \mathrm{~Hz}$. Using a laser interferometer or cosine interferometer to measure the linear displacement error in the system can be measured with a laser. Compared with the measurement data of the grating ruler and the laser interferometer displacement measurement system, the moving stage moves back and forth at intervals of $(-5 \mathrm{~mm}, 10 \mathrm{~mm})$, the absolute value of the difference between the measurement data (Dev) is less than $50 \mathrm{~nm}$, and the measurement time is less than $0.3 \mathrm{~s}$. The study found that compared with the traditional laser interferometer displacement measurement system, the measurement speed of the CPLD-based nanoscale grating displacement measurement system has increased by $27.3 \%$. The specific data is shown in Figure 1.

As can be seen from Figure 1, compared to the traditional laser interferometer displacement measurement system, the measurement speed of the CPLD-based nanoscale grating displacement measurement system is increased by $27.3 \%$

The calculation result of the CPLD-based nanoscale displacement measurement system is calculated through the formula. The zero position $L$ of the nanoscale displacement sensor is $85.3 \mathrm{~mm}$, and the measured displacement value can be calculated. According to the four sets of measurement results measured at the same fixed position, the effective display digits are $0.005 \mathrm{~mm}$, and the displacement resolution reaches $0.2 \mathrm{~mm}$, which meets the precision requirements of the nanograting sensor. Therefore, the displacement measurement system based on the CPLD-based nanoscale grating ruler can effectively improve the displacement measurement accuracy of the sensor and improve the measurement accuracy by $24.7 \%$. Other relevant data are shown in Figure 2.

It can be seen from Figure 2 that the CPLD-based displacement measurement system of the nanoscale grating ruler can effectively improve the displacement measurement

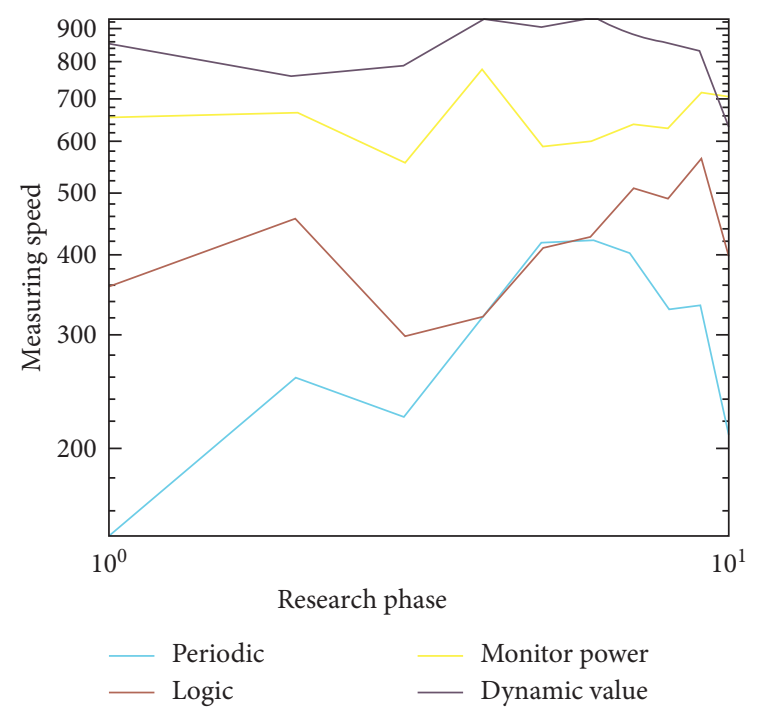

Figure 1: CPLD-based nanoscale grating ruler displacement measurement system increases measurement speed.

accuracy of the sensor, and the measurement accuracy is improved by $24.7 \%$.

7.2. Statistical Results of Displacement Measurement Speed and Accuracy of Nanoscale Grating Ruler. In order to check the measurement effect of the CPLD-based nanoscale displacement measurement system, a high-precision displacement measurement system based on CPLD is designed in this paper. The CPLD design produces a stable excitation pulse L1, which is used for displacement measurement directly with the induction pulse L2 in the CPLD. CPLD smoothens the displacement measurement data by counting data processing, eliminating random errors caused by the high-frequency crystal oscillator, and the corresponding displacement resolution reaches $0.05 \mathrm{~mm}$. The research results show that the CPLD-based nanoscale displacement measurement system simplifies the design of the entire system compared to the traditional displacement measurement circuit implemented by discrete components, 


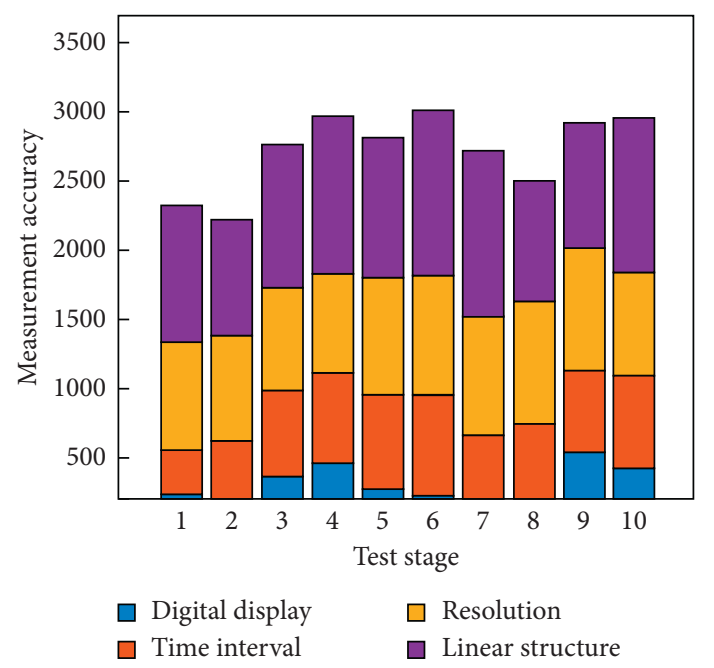

FIGURE 2: Effective improvement of the sensor's displacement measurement accuracy.

which can effectively improve the measurement accuracy of the system and the measurement system. Flexibility and stability improve the robustness of the measurement system by $18.6 \%$. The specific data is shown in Figure 3.

It can be seen from Figure 3 that comparing the CPLDbased nanoscale displacement measurement system to the traditional displacement measurement circuit implemented by discrete components, the system robustness increased by $18.6 \%$.

The experimental results show that from the data analysis of the small-scale microdisplacement model measurement simulation experiment system, it can be clearly seen that the deviation between the average value of the straight line and the simulated reference value of the small-displacement simulation measurement experiment system based on the precision nanometer of CPLD and the advanced rectangular grating scale is, respectively, between 108 and $261 \mathrm{~nm}$, the average deviation of the straight line from the mixed value with the analog value is between 98 and $197 \mathrm{~nm}$, the average value is much smaller than the measurement accuracy of the traditional capacitor displacement measurement sensor, and the average value measured by the system is credible. It can be clearly seen from the data analysis of the large-displacement motion measurement technology laboratory that the largedisplacement motion measuring instrument based on maple's new nano-quality micro-grating ruler has the largest average value of the displacement measurement within a certain period of time for each large-displacement motion measurement of the system. The difference between the value and the minimum measured value distribution should not be less than $40 \mathrm{~nm}$, the distribution is relatively concentrated, the variance is small, and the stability is relatively good. The two displacement measurement values are in the range of $9.62-10.37 \mathrm{~nm}$ and $19.5-22.3 \mathrm{~nm}$, and the measurement values are reliable. The research found that the diffraction efficiency of the nanoscale grating ruler in the plane of the nanoscale grating ruler varies greatly. The rate of change of the diffraction efficiency of the nanoscale grating ruler is related to the grating density. The relevant data is shown in Figure 4.

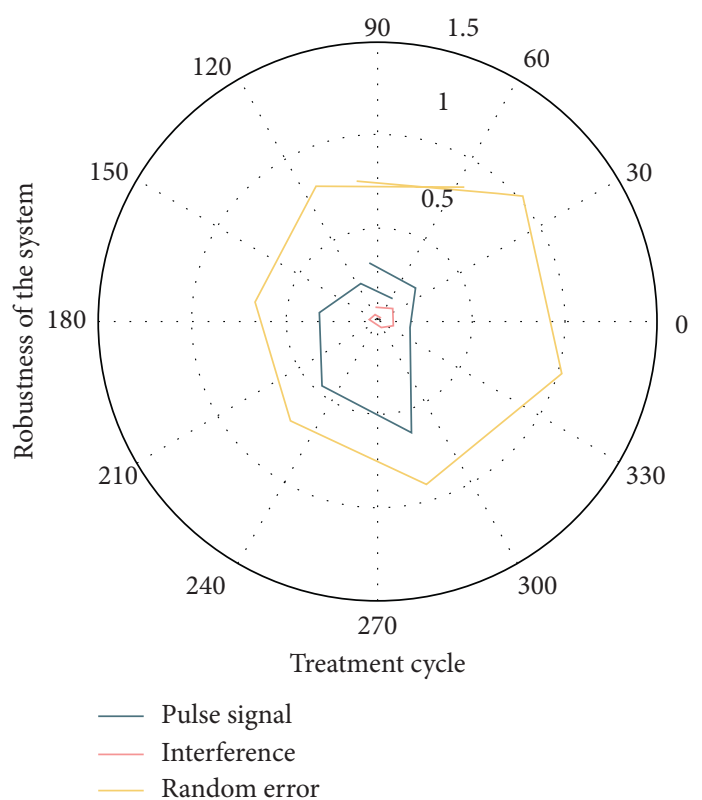

FIgURE 3: Improvement of the flexibility and stability of the measurement system.

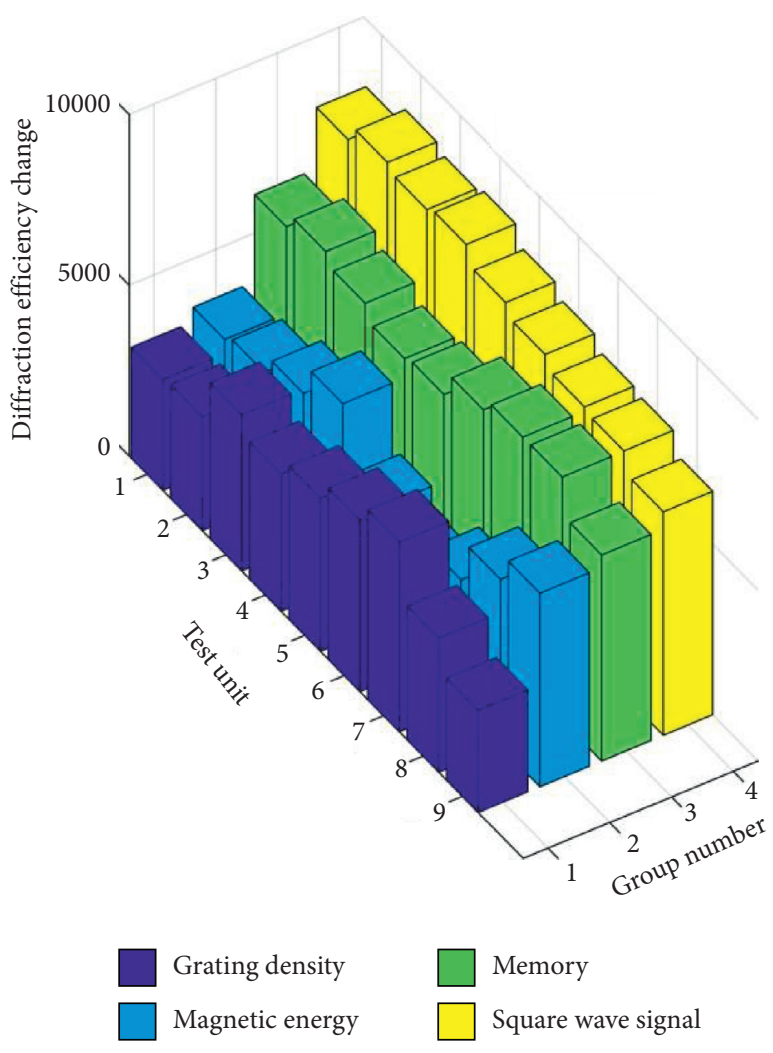

Figure 4: Rate of change of diffraction efficiency of nanoscale.

It can be seen from Figure 4 that the change rate of the diffraction efficiency of the nanoscale grating in the nanoscale grating ruler surface is $0.165 \% \mathrm{~nm}$, and the change rate of the diffraction efficiency of the out-of-plane detection nanograting can reach $0.483 \% \mathrm{~nm}$. 


\section{Conclusions}

Nanoscale grating ruler has an important application as a precision sensor in displacement measurement systems. Traditional displacement measurement systems such as laser interferometric distance measurement system and acoustic wave distance measurement system have been unable to meet the ever-changing requirements of high-precision production technology. Appearing to fill the gap in this field, CPLD is a commonly used programming control system that can achieve high-precision control of instruments and equipment. Based on nanotechnology and CPLD, a new displacement measurement technology came into being. This is the CPLD-based displacement measurement of nanoscale grating rulers system. The research results show that the CPLD-based displacement motion measurement and control system with nanometer-scale precision grating size mainly has three characteristics: high measurement accuracy, strong anti-interference ability, and high measurement motion efficiency, which can effectively meet the requirements of high-precision manufacturing processes and scale displacement motion measurement system requirements.

\section{Data Availability}

No data were used to support the study.

\section{Conflicts of Interest}

The authors declare that they have no conflicts of interest regarding the publication of this study.

\section{Acknowledgments}

This work was supported by the National Key Research and Development Project, Integrated Test Technology and Application for Large Aircraft Assembly Intelligent Equipment (no.2019YFB1707505), and Scientific and Technological Developing Scheme of Jilin Province, "Research on Laser Processing Technology and Equipment of Aviation Wire Harness Terminal" (no. 20200401139GX).

\section{References}

[1] Y. Lu, C. Wei, W. Jia et al., "Two-degree-freedom displacement measurement based on a short period grating in symmetric littrow configuration," Optics Communications, vol. 380, no. 6, pp. 382-386, 2016.

[2] L.-J. Wang, M. Zhang, and Y. Zhu, "A displacement measurement system for ultra-precision heterodyne littrow grating interferometer," Guangxue Jingmi Gongcheng/Optics and Precision Engineering, vol. 25, no. 12, pp. 2975-2985, 2017.

[3] Q. Lv, W. H. Li, Bayanheshig et al., "Interferometric precision displacement measurement system based on diffraction grating," Chinese Optics, vol. 10, no. 1, pp. 39-50, 2017.

[4] Z. Zhang, M. Zhang, and Q. Zhao, "A simplified analysis for deformation behavior of buried pipelines considering disturbance effects of underground excavation in soft clays,"
Arabian Journal of Geosciences, vol. 8, no. 10, pp. 7771-7785, 2015.

[5] Y. Shang, C.-F. Li, and X.-R. Fu, "Two generalized conforming quadrilateral mindlin-reissner plate elements based on the displacement function," Finite Elements in Analysis and Design, vol. 99, no. 7, pp. 24-38, 2015.

[6] D. Versino, T. O. Mourad, and F. L. Addessio, "A global-local discontinuous galerkin finite element for finite-deformation analysis of multilayered shells," Computer Methods in Applied Mechanics and Engineering, vol. 283, no. 3, pp. 1401-1424, 2015.

[7] Y. Xu, R. H. Chen, Q. WuCao, and X. Yu, "Synthesis and cyanide anion recognition of a new displacement fluorescence chemosensor based on two-branched aurone," Fibers and Polymers, vol. 17, no. 2, pp. 181-185, 2016.

[8] W. Peipei, L. Xi, Q. Decheng et al., "Two-dimensional displacement measurement based on two parallel gratings," Review of Scientific Instruments, vol. 89, no. 6, pp. 65-105, 2018.

[9] S. Pinna, S. Melo, F. Laghezza et al., "Photonics-based radar for sub-mm displacement sensing," IEEE Journal of Selected Topics in Quantum Electronics, vol. 23, no. 2, pp. 168-175, 2017.

[10] J. Wang, M. Pierce, A. Donnellan, and J. Parker, "Web services for dynamic coloring of UAVSAR images," Pure and Applied Geophysics, vol. 172, no. 8, pp. 2325-2332, 2015.

[11] Y. O. U. Wei-wei, M. A. O. Jian-liang, and Y. E. Hua, "Design of TFT-LCD display controller based on STM32 and CPLD," Chinese Journal of Liquid Crystals \& Displays, vol. 30, no. 3, pp. 444-450, 2015.

[12] T. J. Ma, W. Liu, and W. Liao, "A study of signal collecting \& processing and display based on CPLD for multiple nuclear pulses," Hedianzixue Yu Tance Jishu/Nuclear Electronics \& Detection Technology, vol. 38, no. 5, pp. 725-729, 2018.

[13] H. Chen, Z. Du, and X. Liu, "A new method for observing the bifurcation of a nonlinear system based on CPLD," Journal of Information Hiding and Multimedia Signal Processing, vol. 8, no. 5, pp. 1141-1148, 2017.

[14] P. Z. Wieczorek, "Lightweight TRNG based on multiphase timing of bistables," IEEE Transactions on Circuits and Systems I: Regular Papers, vol. 63, no. 7, pp. 1043-1054, 2016.

[15] L. Hui, L. Xingqiao, and L. Jing, "CMAC-ADRC algorithm based on adaptive parameter identification for asynchronous motor speed control system," Transactions of the Chinese Society for Agricultural Machinery, vol. 46, no. 3, pp. 358-365, 2015.

[16] L.-H. Wu, X.-Z. Zhang, X.-L. Pan, and M.-Y. Wu, "Design of an static reconfiguration based on FPGA system," International Journal of Multimedia and Ubiquitous Engineering, vol. 11, no. 2, pp. 99-106, 2016.

[17] G. L. Jian, D. W. Ji, B. H. Wen, and F. W. Zhao, "Research on the neutral-point voltage balance for NPC three-level inverters under non-ideal grid conditions," Energies, vol. 11, no. 6, pp. 1331-1135, 2018.

[18] S. Li, H. Wang, Y. Li, Q. Li, B. Zhang, and H. Zhu, "A new mini-grating absolute displacement measuring system for static and dynamic geomechanical model tests," Measurement, vol. 105, no. 5, pp. 25-33, 2017.

[19] G. H. Yuan and N. I. Zheludev, "Detecting nanometric displacements with optical ruler metrology," Science, vol. 364, no. 6442, pp. 771-775, 2019.

[20] J. Lu, X. H. Chen, and Q. F. Tang, "Variable coupling time grating sensor and effects of sensor-head attitudes on 
measuring errors," Guangxue Jingmi Gongcheng/Optics and Precision Engineering, vol. 24, no. 9, pp. 2271-2282, 2016.

[21] X. R. Xiao, M. X. Wang, H. Liang, Q. Gong, and L.-Y. Peng, "Proposal for measuring electron displacement induced by a short laser pulse," Physical Review Letters, vol. 122, no. 5, pp. 1-6, 2019.

[22] G. Zhu, C. Zhang, and M. Fu, "Development of alternating light field space-time coupling type displacement measuring system," Guangxue Jingmi Gongcheng/Optics and Precision Engineering, vol. 25, no. 8, pp. 2011-2022, 2017.

[23] K. Liu, M. Sun, Y. Wu, and T. Zhu, "Comparison of accuracy stability using a thermal compensator and grating ruler," Journal of the Brazilian Society of Mechanical Sciences and Engineering, vol. 38, no. 8, pp. 2403-2411, 2016.

[24] J. Yang and J. Zhang, "Automatic verification system for time grating angular displacement sensors," Zhongguo Jixie Gongcheng/China Mechanical Engineering, vol. 26, no. 14, pp. 1932-1937, 2015.

[25] S Sun, S. C. Li, L. P. Li et al., "Design of a displacement monitoring system based on optical grating and numerical verification in geomechanical model test of water leakage of tunnel," Geotechnical \& Geological Engineering, vol. 36, no. 22, pp. 1-12, 2018. 